BULL. AUSTRAL. MATH. SOC.

\title{
A dimension formula relating to algebraic groups
}

\section{Su-shing Chen}

\begin{abstract}
An upper bound is given of the dimension of certain spaces of cusp harmonic forms of arithmetic subgroups $\Gamma$ of semisimple algebraic groups $G$ in terms of the multiplicities of corresponding irreducible unitary representations of the group $G_{R}$ of real rational points of $G$ in the space ${ }^{\circ} L^{2}\left(G_{R} / \Gamma\right)$ of cusp forms.
\end{abstract}

\section{Introduction}

Our formula can be considered to be related to the duality of Gel'fand and Pyateckii-Shapiro of a discrete subgroup $\Gamma$ of $G_{R}$ such that $G_{R} / \Gamma$ is compact [3]. An essential point of the duality in [3] is that $L^{2}\left(G_{R} / \Gamma\right)$ is a countable direct sum of irreducible unitary representations of $G_{\mathrm{R}}$. If $G_{\mathrm{R}} / \Gamma$ is not compact, then $L^{2}\left(G_{\mathrm{R}} / \Gamma\right)$ contains continuous and discrete spectrum in general. However, the closed invariant subspace ${ }^{\circ} L^{2}\left(G_{\mathrm{R}} / \Gamma\right)$ of $L^{2}\left(G_{\mathrm{R}} / \Gamma\right)$ is still a countable direct sum of irreducible unitary representations of $G_{R}$ for an arithmetic subgroup $\Gamma$ of $G_{R}$ [4]. Consequently, we can obtain an upper bound of the Garland space of cusp harmonic forms which is a closed invariant subspace of the space studied in [2] by Garland. For basic definitions and facts about algebraic groups and their arithmetic subgroups, we refer to $[1]$.

Received 10 November 1970. 


\section{The formula}

We assume that $G$ is a connected semisimple linear algebraic group which is defined and simple over $Q$. Moreover, we assume that $G$ has Q-rank 1 , that is, $\operatorname{dim} S_{Q}=1$, where $S_{Q}$ is a maximal Q-split torus of $G$. Let $G_{R}$ denote the Lie algebra of $G_{R}$ and $\underline{\underline{G}}$ denote the complexification of $\underline{G}_{R}$. Denote by $\Delta_{G}$ the Casimir operator which is a unique element of the center of the universal enveloping algebra of $\underline{G}$.

The space ${ }^{\circ} L^{2}\left(G_{\mathrm{R}} / \Gamma\right)$ of cusp forms consists of elements of $L^{2}\left(G_{\mathrm{R}} / \Gamma\right)$ satisfying

$$
\int_{U_{\mathrm{R}} / U_{\mathrm{R}} \cap \Gamma} f(x u) d u=0
$$

for almost all $x \in G_{\mathrm{R}}$, where $U$ is the unipotent radical of an arbitrary parabolic subgroup $P$ of $G$.

We fix a certain maximal compact subgroup $K \subset G_{R}$. Let $V$ be a finite dimensional complex vector space with a positive definite hermitian inner product. Then let $\sigma: K+$ Aut $V$ be a representation of $K$ which is unitary with respect to the given inner product. We let $d_{\sigma}$ denote the complex dimension of $V$ and let $\xi_{\sigma}$ denote the character of $\sigma$. Let $d k$ denote the Haar measure on $K$ normalized so that $\int_{K} d k=1$.

For $\nu \in C$, the Garland space $G(\sigma, \nu)$ of harmonic forms is defined by

$$
\begin{aligned}
& G(\sigma, \nu)=\left\{f \in L^{2}\left(G_{\mathrm{R}} / \Gamma\right) \cap C^{\infty}\left(G_{\mathrm{R}} / \Gamma\right) \mid \Delta_{G} f=\nu f,\right. \\
&\left.d_{\sigma} \int_{K} \xi_{\sigma}(k) f\left(k^{-1} x\right) d k=f(x), x \in G_{\mathrm{R}} / \Gamma\right\} .
\end{aligned}
$$

If $G(\sigma, \nu) \neq 0$ and $G$ has $Q$-rank $I$, then $\nu$ is real and

$\operatorname{dim} G(\sigma, \nu)<\infty$ ([2]). The Garland space ${ }^{\circ} G(\sigma, \nu)$ of cusp harmonic forms is defined by

$$
{ }^{o} G(\sigma, \nu)=\left\{f \in G(\sigma, v) \cap{ }^{\circ} L^{2}\left(G_{\mathrm{R}} / \Gamma\right)\right\}
$$


Let $\hat{G}_{\mathrm{R}}$ denote the set of irreducible unitary representations $\pi$ of $G_{\mathrm{R}}$. Let $H_{\pi}$ be the representation space of $\pi, m(\pi)$ be the multiplicity of $\pi$ in ${ }^{0} L^{2}\left(G_{R} / \Gamma\right)$ and $\Delta_{\pi}$ be the Casimir operator of the representation $\pi$. Since $\pi$ is irreducible, there exists a complex number $\nu_{\pi}$ such that $\Delta_{\pi} \varphi=\nu_{\pi} \varphi$ for $\varphi$ in the domain of $\Delta_{\pi}$, which is dense in $H_{\pi}$. Let $\hat{G}_{\mathrm{R}}(v)$ denote the set of irreducible unitary representations $\pi$ of $G_{R}$ such that $\Delta_{\pi}=\nu_{\pi} \cdot 1$ and $\nu_{\pi}=\nu$. Fix an irreducible unitary representation $\pi$ and its representation space $H_{\pi}$. For any irreducible unitary representation $\sigma$ of $K$, we define a linear transformation $E_{\sigma}$ in $H_{\pi}$ by

$$
E_{\sigma} v=d_{\sigma} \int_{K} \xi_{\sigma}(k) \pi\left(k^{-1}\right) v d k
$$

for $v \in H_{\pi}$. Then $E_{\sigma}$ is a continuous projection. We let $H_{\pi, \sigma}=E_{\sigma}\left(H_{\pi}\right)$. The dimension of $H_{\pi, \sigma}$ is finite dimensional and is denoted by $d\left(H_{\pi, \sigma}\right)$.

We write ${ }^{\circ} L^{2}\left(G_{\mathrm{R}} / \Gamma\right)=\sum_{i=1}^{\infty} \oplus H_{i}$, where $H_{i}$ is the representation space of the irreducible unitary representation $\pi_{i}$. Note that, for $\varphi \in C^{\infty}\left(G_{\mathrm{R}} / \Gamma\right) \cap{ }^{\circ} L^{2}\left(G_{\mathrm{R}} / \Gamma\right)$, the regular representation $\lambda$ of $G_{\mathrm{R}}$ on ${ }^{\circ} L^{2}\left(G_{R} / \Gamma\right)$ satisfies $\Delta_{\lambda} \varphi=\Delta_{G} \varphi$. This follows from an easy computation.

For any $f \in{ }^{\circ} G(\sigma, v), f \in{ }^{o} L^{2}\left(G_{R} / \Gamma\right) \cap C^{\infty}\left(G_{R} / \Gamma\right), \Delta_{G} f=\nu f$ and $E_{\sigma} f=f$. Let $P_{i}$ be the projection of ${ }^{\circ} L^{2}\left(G_{R} / \Gamma\right)$ onto $H_{i}$. Since $f$ is differentiable, for each $X \in \mathrm{G}_{\mathrm{R}}$, 


$$
\begin{aligned}
P_{i} \lambda(X) f & =\lim _{t \rightarrow 0} \frac{1}{t} P_{i}(\lambda(\exp t X) f-f) \\
& =\lim _{t \rightarrow 0} \frac{1}{t}\left(\lambda(\exp t X) P_{i} f-P_{i} f\right) \\
& =\lim _{t \rightarrow 0} \frac{1}{t}\left(\pi_{i}(\exp t X) P_{i} f-P_{i} f\right) \\
& =\pi_{i}(X) P_{i} f .
\end{aligned}
$$

Hence $P_{i} f$ is in the domain of $\Delta_{\pi_{i}}, P_{i} \Delta_{\lambda} f=\Delta_{\pi_{i}} P_{i} f$ and $\Delta_{\pi_{i}} P_{i} f=\lambda_{\pi_{i}} P_{i} f$. If $\pi_{i} \neq \hat{G}_{R}(\nu)$, then $P_{i} f=0$. Hence $f=P_{i} f+\ldots+P_{i} f$. Since $E_{\sigma} \cdot P_{i}=P_{i} \cdot E_{\sigma}, P_{i} f \in H_{i, \sigma}$ for $i \in\left\{i_{1}, \ldots, i_{t}\right\}$. Consequently, we get the following

THEOREM. Let $G$ be a connected semisimple linear algebraic group. which is defined and simple over Q. We assume that $G$ has Q-rank 1 . Then

$$
\operatorname{dim}^{\circ} G(\sigma, \nu) \leq \sum_{\pi \in \hat{G}_{\mathrm{R}}(\nu)} m(\pi) d\left(H_{\pi, \sigma}\right)
$$

\section{References}

[1] Armand Borel, "Linear algebraic groups", Algebraic groups and discontinuous subgroups (Proc. Sympos. Pure Math., Boulder, Colo., 1965), 3-19. (Amer. Math. Soc., Providence, Rhode Island, 1966.)

[2] Howard Garland, "The spectrum of noncompact $G / \Gamma$ and the cohomology of arithmetic groups", Buz2. Amer. Math. Soc. 75 (1969), 807-811.

[3] I.M. Gel'fand and 1.1. PyateckiT-Sapiro, "Theory of representations and theory of automorphic functions", Amer. Math. Soc. Trans 2. (2) 26 (1963), 173-200. 
[4] R. Godement, "The spectral decomposition of cusp-forms", Algebraic groups and discontinuous subgroups (Proc. Sympos. Pure Math., Boulder, Colo., 1965), 225-234. (Amer. Math. Soc., Providence, Rhode Island, 1966).

University of Florida,

Gainesville,

Florida, USA. 